\title{
Rota-Baxter bialgebra structures arising from (co-)quasi-idempotent elements
}

\author{
Tianshui Ma*(D), Jie Li (iD, Haiyan Yang (iD \\ School of Mathematics and Information Science, Henan Normal University, Xinxiang 453007, China
}

\begin{abstract}
In this note, we construct Rota-Baxter (coalgebras) bialgebras by (co-)quasi-idempotent elements and prove that every finite dimensional Hopf algebra admits nontrivial RotaBaxter bialgebra structures and tridendriform bialgebra structures. We give all the forms of (co)-quasi-idempotent elements and related structures of tridendriform (co, bi)algebras and Rota-Baxter (co, bi)algebras on the well-known Sweedler's four-dimensional Hopf algebra.
\end{abstract}

Mathematics Subject Classification (2020). 16W99, 16T05

Keywords. Rota-Baxter bialgebras, (co-)quasi-idempotent element, tridendriform bialgebra

\section{Introduction}

Rota-Baxter algebras were introduced in [11] in the context of differential operators on commutative Banach algebras and since [1], intensively studied in probability and combinatorics, and more recently in mathematical physics, such as free Rota-Baxter algebras, Lie algebras, multiple zeta values, differential algebras and Connes-Kreimer renormalization theory in quantum field theory, see ([2-7], etc.). One can refer to the book [2] for the detailed theory of Rota-Baxter algebras.

In 2014, based on the dual method in the Hopf algebra theory, Jian and Zhang in [8] defined the notion of Rota-Baxter coalgebras and also provided various examples of the new object. Then Rota-Baxter bialgebras were presented in [9] whose examples can be constructed from the well-known Radford biproduct. In 2017, Jian construct quasiidempotent Rota-Baxter operators by quasi-idempotent elements and show that every finite dimensional Hopf algebra admits nontrivial Rota-Baxter algebra structures and tridendriform algebra structures (see [7]).

So it is natural to consider if every finite dimensional Hopf algebra admits nontrivial Rota-Baxter bialgebra structure and tridendriform bialgebra structure. In this paper, we give a positive answer to this question. This is the motivation to write this paper.

This paper is organized as follows. In Section 2, we list some definitions that will be used later. In Section 3, we present the notions of tridendriform coalgebras, tridendriform

\footnotetext{
*Corresponding Author.

Email addresses: matianshui@yahoo.com (T. Ma), lijie_0224@163.com (J. Li), yhy3023551288@163.com (H. Yang)

Received: 06.02.2020; Accepted: 29.05.2020
} 
bialgebras, and co-quasi-idempotent element in a coalgebra. We use (co-)quasi-idempotent element to construct Rota-Baxter coalgebras and bialgebras. And then we prove that every finite dimensional Hopf algebra admits nontrivial Rota-Baxter bialgebra structures and tridendriform bialgebra structures. All the forms of (co)-quasi-idempotent elements and related structures of tridendriform (co, bi)algebras and Rota-Baxter (co, bi)algebras on the well-known Sweedler's four-dimensional Hopf algebra are provided in Section 4.

\section{Preliminaries}

For simplicity, we fix our ground field to be the complex number field $\mathbb{C}$ throughout this paper. All the objects we discuss are defined over $\mathbb{C}$ unless otherwise specified. For an algebra $A$, we denote its multiplication $\mu_{A}$ (or simply $\mu$ ) by $\mu_{A}(a \otimes b)=a b$.

In what follows, we recall some useful definitions which will be used later (see $[2,7,9]$ ).

Definition 2.1. For $\lambda \in \mathbb{C}$, a Rota-Baxter algebra of weight $\lambda$ is an associative algebra $A$ together with a linear map $R: A \longrightarrow A$ such that

$$
R(a) R(b)=R(a R(b))+R(R(a) b)+\lambda R(a b)
$$

for all $a, b \in A$. Such a linear operator is called a Rota-Baxter operator of weight $\lambda$ on $A$.

Remark 2.2. If $R$ is a Rota-Baxter operator of weight 1 , then $\lambda R$ is a Rota-Baxter operator of weight $\lambda$. Conversely, if $R$ is a Rota-Baxter operator of weight $\lambda$ and $\lambda$ is invertible, then $\lambda^{-1} R$ is a Rota-Baxter operator of weight 1 .

Definition 2.3. Let $C$ be a vector space and $\Delta_{C}: C \longrightarrow C \otimes C$ (here we use Sweedler's notation and denote $\Delta_{C}(c)$ by $\left.c_{1} \otimes c_{2}\right), \varepsilon_{C}: C \longrightarrow \mathbb{C}$ two linear maps. Then $C$ is a coassociative coalgebra if

$$
c_{11} \otimes c_{12} \otimes c_{2}=c_{1} \otimes c_{21} \otimes c_{22} \text { and } \varepsilon_{C}\left(c_{1}\right) c_{2}=c_{1} \varepsilon_{C}\left(c_{2}\right)=c
$$

hold for all $c \in C$.

Let $\gamma$ be an element in $\mathbb{C}$. A pair $(C, Q)$ is called a Rota-Baxter coalgebra of weight $\gamma$ if $C$ is a coassociative coalgebra and $Q$ is a linear endomorphism of $C$ satisfying that for all $c \in C$,

$$
Q\left(c_{1}\right) \otimes Q\left(c_{2}\right)=Q(c)_{1} \otimes Q\left(Q(c)_{2}\right)+Q\left(Q(c)_{1}\right) \otimes Q(c)_{2}+\gamma Q(c)_{1} \otimes Q(c)_{2} .
$$

The map $Q$ is called a Rota-Baxter operator weight $\gamma$ on $C$.

Remark 2.4. If $Q$ is a Rota-Baxter operator of weight 1 , then $\gamma Q$ is a Rota-Baxter operator of weight $\gamma$. Conversely, if $Q$ is a Rota-Baxter operator of weight $\gamma$ and $\gamma$ is invertible, then $\gamma^{-1} Q$ is a Rota-Baxter operator of weight 1 .

Definition 2.5. Let $H$ be a vector space. $H$ is a bialgebra if $\left(H, \mu_{H}\right)$ is an associative algebra and $\left(H, \Delta_{H}\right)$ is a coassociative coalgebra such that $\Delta_{H}$ and $\varepsilon_{H}$ are algebra maps.

Let $\lambda, \gamma$ be elements in $\mathbb{C}$ and $H$ a bialgebra (maybe without unit and counit). A triple $(H, R, Q)$ is called a Rota-Baxter bialgebra of weight $(\lambda, \gamma)$ if $(H, R)$ is a Rota-Baxter algebra of weight $\lambda$ and $(H, Q)$ is a Rota-Baxter coalgebra of weight $\gamma$.

Remark 2.6. If $(H, R, Q)$ is a Rota-Baxter bialgebra of weight $(1,1)$, then $(H, \lambda R, \gamma Q)$ is a Rota-Baxter bialgebra of weight $(\lambda, \gamma)$. Conversely, if $(H, R, Q)$ is a Rota-Baxter bialgebra of weight $(\lambda, \gamma)$ and $\lambda, \gamma$ are invertible, then $\left(H, \lambda^{-1} R, \gamma^{-1} Q\right)$ is a Rota-Baxter bialgebra of weight $(1,1)$.

Definition 2.7. Let $A$ be an associative algebra and $\lambda \in \mathbb{C}$. A linear endomorphism $\phi$ of $A$ is called a quasi-idempotent operator of weight $\lambda$ on $A$ if $\phi^{2}=-\lambda \phi$. A nonzero element $\xi \in A$ is called a quasi-idempotent element of weight $\lambda$ if $\xi^{2}=-\lambda \xi$. 
Definition 2.8. Let $V$ be a vector space, and $\prec, \succ, \cdot: V \otimes V \longrightarrow V$ be three linear maps. The quadruple $(V, \prec, \succ, \cdot)$ is called a tridendriform algebra if the following conditions are satisfied: for all $x, y, z \in V$,

$$
\begin{aligned}
& (x \prec y) \prec z=x \prec(y * z), \quad(x \succ y) \prec z=x \succ(y \prec z), \\
& (x * y) \succ z=x \succ(y \succ z), \quad(x \succ y) \cdot z=x \succ(y \cdot z), \\
& (x \prec y) \cdot z=x \cdot(y \succ z), \quad(x \cdot y) \prec z=x \cdot(y \prec z), \quad(x \cdot y) \cdot z=x \cdot(y \cdot z),
\end{aligned}
$$

where $x * y=x \prec y+x \succ y+x \cdot y$.

Remark 2.9. Given a Rota-Baxter algebra $(A, R)$ of weight 1 , we define

$$
a \prec b=a \cdot R(b), \quad a \succ b=R(a) \cdot b,
$$

for all $a, b \in A$. Then $\left(V, \prec, \succ, \mu_{A}\right)$ is a tridendriform algebra.

\section{Construction of tridendriform co(bi)algebra and Rota-Baxter bialge- bras}

In this section, based on the dual method in Hopf algebra theory, we define tridendriform co(bi)algebras, co-quasi-idempotent elements, then construct tridendriform co(bi)algebras and Rota-Baxter co(bi)algebras through (co-)quasi-idempotent elements.

Definition 3.1. Let $V$ be a vector space, and $\Delta_{\prec}, \Delta_{\succ}, \Delta$. $: V \longrightarrow V \otimes V$ be three linear maps (write $\Delta_{\prec}(x)=x^{1} \otimes x^{2}, \Delta_{\succ}(x)=x^{(1)} \otimes x^{(2)}, \Delta .(x)=x^{[1]} \otimes x^{[2]}$ ). The quadruple $\left(V, \Delta_{\prec}, \Delta_{\succ}, \Delta\right.$.) is called a tridendriform coalgebra if the following conditions are satisfied: for all $x \in V$,

$$
\begin{gathered}
x^{11} \otimes x^{12} \otimes x^{2}=x^{1} \otimes\left(x^{21} \otimes x^{22}+x^{2(1)} \otimes x^{2(2)}+x^{2[1]} \otimes x^{2[2]}\right), \\
x^{1(1)} \otimes x^{1(2)} \otimes x^{2}=x^{(1)} \otimes x^{(2) 1} \otimes x^{(2) 2}, \\
\left(x^{(1) 1} \otimes x^{(1) 2}+x^{(1)(1)} \otimes x^{(1)(2)}+x^{(1)[1]} \otimes x^{(1)[2]}\right) \otimes x^{(2)}=x^{(1)} \otimes x^{(2)(1)} \otimes x^{(2)(2)}, \\
x^{[1](1)} \otimes x^{[1](2)} \otimes x^{[2]}=x^{(1)} \otimes x^{(2)[1]} \otimes x^{(2)[2]}, \\
x^{[1] 1} \otimes x^{[1] 2} \otimes x^{[2]}=x^{[1]} \otimes x^{[2](1)} \otimes x^{[2](2)}, \\
x^{1[1]} \otimes x^{1[2]} \otimes x^{2}=x^{[1]} \otimes x^{[2] 1} \otimes x^{[2] 2}, \\
x^{[1][1]} \otimes x^{[1][2]} \otimes x^{[2]}=x^{[1]} \otimes x^{[2][1]} \otimes x^{[2][2]} .
\end{gathered}
$$

Rota-Baxter coalgebras are closely related to tridendriform coalgebras.

Lemma 3.2. Given a Rota-Baxter coalgebra $(C, Q)$ of weight 1 , we define

$$
\Delta_{\prec}(c)=c_{1} \otimes Q\left(c_{2}\right), \quad \Delta_{\succ}(c)=Q\left(c_{1}\right) \otimes c_{2} .
$$

Then $\left(C, \Delta_{\prec}, \Delta_{\succ}, \Delta_{C}\right)$ is a tridendriform coalgebra.

Proof. It can be proved by direct computation.

Definition 3.3. Let $V$ be a vector space. A seven-tuple $\left(V, \prec, \succ, \cdot, \Delta_{\prec}, \Delta_{\succ}, \Delta\right.$.) is called a tridendriform bialgebra if $(V, \prec, \succ, \cdot)$ is a tridendriform algebra and at the same time $\left(V, \Delta_{\prec}, \Delta_{\succ}, \Delta\right.$. $)$ is a tridendriform coalgebra.

Proposition 3.4. Let $H$ be a bialgebra and $(H, R, Q)$ a Rota-Baxter bialgebra of weight $(1,1)$. Define

$$
\begin{aligned}
& x \prec y=x R(y), \quad x \succ y=R(x) y, \\
& \Delta_{\prec}(x)=x_{1} \otimes Q\left(x_{2}\right), \quad \Delta_{\succ}(x)=Q\left(x_{1}\right) \otimes x_{2},
\end{aligned}
$$

for all $x, y \in H$. Then $\left(V, \prec, \succ, \mu_{H}, \Delta_{\prec}, \Delta_{\succ}, \Delta_{H}\right)$ is a tridendriform bialgebra.

Proof. It is a consequence of Lemma 3.2 and the Remark 2.9. 
Definition 3.5. Let $C$ be a coassociative coalgebra and $\gamma \in \mathbb{C}$. A linear endomorphism $\vartheta$ of $C$ is called a quasi-idempotent operator of weight $\gamma$ on $C$ if $\vartheta^{2}=-\gamma \vartheta$. A nonzero element $\tau \in C^{*}$ is called a co-quasi-idempotent element of weight $\gamma$ if $\tau\left(c_{1}\right) \tau\left(c_{2}\right)=-\gamma \tau(c)$ for all $c \in C$.

Proposition 3.6. Let $C$ be a coalgebra. Given a co-quasi-idempotent element $\tau \in C^{*}$ of weight $\gamma \neq 0$. Three linear maps $\Delta_{\prec}, \Delta_{\succ}, \Delta$. :C $\longrightarrow C \otimes C$ defined below endow a tridendriform coalgebra structure on $C$ : for all $c \in C$,

$$
\Delta_{\prec}(c)=\gamma^{-1} c_{1} \otimes \tau\left(c_{2}\right) c_{3}, \Delta_{\succ}(c)=\gamma^{-1} \tau\left(c_{1}\right) c_{2} \otimes c_{3}, \Delta .(c)=c_{1} \otimes c_{2} .
$$

Proof. We only check the first equality in the definition of tridendreform coalgebra as follows. For all $c \in C$, we can get

$$
\begin{aligned}
c^{1} \otimes & \left(c^{21} \otimes c^{22}+c^{2(1)} \otimes c^{2(2)}+c^{2[1]} \otimes c^{2[2]}\right) \\
= & \gamma^{-2} c_{1} \tau\left(c_{2}\right) \tau\left(c_{32}\right) \otimes c_{31} \otimes c_{33}+\gamma^{-2} c_{1} \tau\left(c_{2}\right) \tau\left(c_{31}\right) \otimes c_{32} \otimes c_{33} \\
& +\gamma^{-1} c_{1} \tau\left(c_{2}\right) \otimes c_{31} \otimes c_{32} \\
= & \gamma^{-2} c_{1} \tau\left(c_{2}\right) \tau\left(c_{32}\right) \otimes c_{31} \otimes c_{33}-\gamma^{-1} c_{1} \tau\left(c_{2}\right) \otimes c_{31} \otimes c_{32} \\
& +\gamma^{-1} c_{1} \tau\left(c_{2}\right) \otimes c_{31} \otimes c_{32} \\
= & \gamma^{-2} c_{1} \tau\left(c_{2}\right) \tau\left(c_{32}\right) \otimes c_{31} \otimes c_{33} \\
= & c^{11} \otimes c^{12} \otimes c^{2}
\end{aligned}
$$

finishing the proof.

Theorem 3.7. Let $H$ be a bialgebra. Given a quasi-idempotent element $\xi \in H$ of weight $\lambda \neq 0$ and a co-quasi-idempotent element $\tau \in H^{*}$ of weight $\gamma \neq 0$. Six linear maps $\prec, \succ, \cdot: H \otimes H \longrightarrow H$ and $\Delta_{\prec}, \Delta_{\succ}, \Delta .: H \longrightarrow H \otimes H$ defined below endow a tridendriform bialgebra structure on $H$ : for all $x, y \in H$,

$$
x \prec y=\lambda^{-1} x \xi y, x \succ y=\lambda^{-1} \xi x y, x \cdot y=x y,
$$

and

$$
\Delta_{\prec}(x)=\gamma^{-1} x_{1} \otimes \tau\left(x_{2}\right) x_{3}, \Delta_{\succ}(x)=\gamma^{-1} \tau\left(x_{1}\right) x_{2} \otimes x_{3}, \Delta .(x)=x_{1} \otimes x_{2} .
$$

Proof. We can finish the proof by [7, Corollary 2.4] and Proposition 3.6.

Now we use co-quasi-idempotent elements to construct quasi-idempotent Rota-Baxter operators.

Proposition 3.8. For a fixed co-quasi-idempotent element $\tau \in C^{*}$ of weight $\gamma$, we define linear map $Q_{\tau}: C \longrightarrow C$ by $Q_{\tau}(c)=\tau\left(c_{1}\right) c_{2}$ for any $c \in C$. Then $Q_{\tau}$ is a quasi-idempotent Rota-Baxter operator of weight $\gamma$ on $C$.

Proof. It is direct to prove that $Q_{\tau}^{2}=-\gamma Q_{\tau}$ by the definition of co-quasi-idempotent element. Next for any $c \in C$, we have

$$
\begin{aligned}
& Q_{\tau}(c)_{1} \otimes Q_{\tau}\left(Q_{\tau}(c)_{2}\right)+Q_{\tau}\left(Q_{\tau}(c)_{1}\right) \otimes Q_{\tau}(c)_{2}+\gamma Q_{\tau}(c)_{1} \otimes Q_{\tau}(c)_{2} \\
& \quad=\tau\left(c_{1}\right) c_{21} \otimes \tau\left(c_{221}\right) c_{222}+\tau\left(c_{1}\right) \tau\left(c_{211}\right) c_{212} \otimes c_{22}+\gamma \tau\left(c_{1}\right) c_{21} \otimes c_{22} \\
& =\tau\left(c_{1}\right) c_{21} \otimes \tau\left(c_{221}\right) c_{222}-\gamma \tau\left(c_{1}\right) c_{21} \otimes c_{22}+\gamma \tau\left(c_{1}\right) c_{21} \otimes c_{22} \\
& =\tau\left(c_{11}\right) c_{12} \otimes \tau\left(c_{21}\right) c_{22} \\
& =Q_{\tau}\left(c_{1}\right) \otimes Q_{\tau}\left(c_{2}\right),
\end{aligned}
$$

finishing the proof.

Theorem 3.9. Let $H$ be a bialgebra. Suppose that $\xi \in H$ is a quasi-idempotent of weight of $\lambda$ and $\tau \in H^{*}$ is a co-quasi-idempotent element of weight $\gamma$, then $\left(H, R_{\xi}, Q_{\tau}\right)$ is a Rota-Baxter bialgebra of weight $(\lambda, \gamma)$, where

$$
R_{\xi}(x)=\xi x, \quad Q_{\tau}(x)=\tau\left(x_{1}\right) x_{2},
$$


for all $x \in H$.

Proof. By [7, Prosition 2.2] and Proposition 3.8, we can finish the proof.

Let recall the following result from [10] on finite dimensional Hopf algebra. As we know, a Hopf algebra $H$ is a bialgebra $H$ with an antipode $S$, where the linear map $S: H \longrightarrow H$ is the convolution inverse of identity map $\operatorname{id}_{H}$ in convolution algebra $\operatorname{Hom}(H, H)$.

Let $H$ be a finite dimensional Hopf algebra. Then there is a unique element $x_{H}$ such that

$$
\left\langle a^{*}, x_{H}\right\rangle=\operatorname{Tr}\left(l_{a^{*}}\right), \forall a^{*} \in H^{*} .
$$

Furthermore, the element $x_{H}$ has the following properties.

$$
\varepsilon\left(x_{H}\right)=\operatorname{dim}(H), \quad x_{H}^{2}=\varepsilon\left(x_{H}\right) x_{H} .
$$

that is to say, $x_{H} \in H$ is a quasi-idempotent element of weight $-\operatorname{dim}(H)$ on $H$.

When $H$ is finite dimensional, $H^{*}$ is also a finite dimensional Hopf algebra and $\operatorname{dim}\left(H^{*}\right)=\operatorname{dim}(H)$. So using the above result to finite dimensional Hopf algebra $H^{*}$, we can get: there is a unique element $\chi_{H} \in H^{*}$ such that

$$
\left\langle\chi_{H}, a\right\rangle=\operatorname{Tr}\left(l_{a}\right), \forall a \in H .
$$

Furthermore, the element $\chi_{H}$ has the following properties.

$$
\begin{aligned}
& \varepsilon_{H^{*}}\left(\chi_{H}\right)=\left\langle\chi_{H}, 1_{H}\right\rangle=\operatorname{dim}(H), \quad \chi_{H}^{2}=\varepsilon_{H^{*}}\left(\chi_{H}\right) \chi_{H} \\
& \text { i.e., } \chi_{H}\left(a_{1}\right) \chi_{H}\left(a_{2}\right)=\left\langle\chi_{H}, 1_{H}\right\rangle \chi_{H}(a)=\operatorname{dim}(H) \chi_{H}(a),
\end{aligned}
$$

that is to say, $\chi \in H^{*}$ is a co-quasi-idempotent element of weight $-\operatorname{dim}(H)$ on $H$.

Also we know the integral $\Lambda$ and cointegral $\Lambda$ (i.e. integral of $H^{*}$ ) for finite dimensional Hopf algebra $H$ must exist, and $\Lambda$ is a quasi-idempotent element and $\Lambda$ is a co-quasiidempotent element.

By combining the discussions above, we see that $R_{x_{H}}, R_{\Lambda}$ and $Q_{\chi}, Q_{\bigwedge}$ are Rota-Baxter operators on $H$. As a consequence, we have

Theorem 3.10. Every finite dimensional Hopf algebra admits nontrivial Rota-Baxter coalgebra and bialgebra structures and tridendriform coalgebra and bialgebra structures.

\section{An example}

The well-known Sweedler's four-dimensional Hopf algebra $H_{4}$ is a very popular example in the theory of Hopf algebras, and many researchers pay their attention to it because there are many nice properties on it. In this section, we will apply the above results in Section 3 to $\mathrm{H}_{4}$, and give all the forms of (co)-quasi-idempotent elements and related structures of tridendriform (co, bi)algebras and Rota-Baxter (co, bi)algebras.

Let $H_{4}$ be the algebra generated by two elements $x$ and $y$ subject to

$$
x^{2}=1, \quad y^{2}=0, \quad y x=-x y .
$$

Then $H_{4}$ is a four-dimensional algebra with a linear basis $\{1, x, y, x y\}$ (see $[10,12]$ ), explicitly, its multiplication is

\begin{tabular}{c|cccc}
$\mu_{H_{4}}$ & 1 & $x$ & $y$ & $x y$ \\
\hline 1 & 1 & $x$ & $y$ & $x y$ \\
$x$ & $x$ & 1 & $x y$ & $y$ \\
$y$ & $y$ & $-x y$ & 0 & 0 \\
$x y$ & $x y$ & $-y$ & 0 & 0
\end{tabular}.


Moreover it is a Hopf algebra equipped with the following operations:

$$
\begin{gathered}
\Delta(x)=x \otimes x, \quad \Delta(y)=1 \otimes y+y \otimes x, \\
\varepsilon(x)=1, \quad \varepsilon(y)=0, \\
S(x)=x, \quad S(y)=x y .
\end{gathered}
$$

Denote by $\left\{f_{1}, f_{2}, f_{3}, f_{4}\right\}$ the dual basis of $\{1, x, y, x y\}$, i.e.,

\begin{tabular}{c|cccc} 
& 1 & $x$ & $y$ & $x y$ \\
\hline$f_{1}$ & 1 & 0 & 0 & 0 \\
$f_{2}$ & 0 & 1 & 0 & 0 \\
$f_{3}$ & 0 & 0 & 1 & 0 \\
$f_{4}$ & 0 & 0 & 0 & 1
\end{tabular}.

Then the multiplication of $H_{4}{ }^{*}$ is

\begin{tabular}{c|cccc}
$\mu_{H_{4}}{ }^{*}$ & $f_{1}$ & $f_{2}$ & $f_{3}$ & $f_{4}$ \\
\hline$f_{1}$ & $f_{1}$ & 0 & $f_{3}$ & 0 \\
$f_{2}$ & 0 & $f_{2}$ & 0 & $f_{4}$ \\
$f_{3}$ & 0 & $f_{3}$ & 0 & 0 \\
$f_{4}$ & $f_{4}$ & 0 & 0 & 0
\end{tabular}

Thus by the definitions of (co-)quasi-idempotent element, we have

\begin{tabular}{c|c|c} 
& quasi-idempotent element $\xi$ & weight $\lambda$ \\
\hline$\xi_{1}$ & $l_{1}(1+x)+l_{2} y+l_{3} x y$ & $-2 l_{1}$ \\
\hline$\xi_{2}$ & $l_{1}(1-x)+l_{2} y+l_{3} x y$ & $-2 l_{1}$ \\
\hline$\xi_{3}$ & $l_{1} 1$ & $-l_{1}$
\end{tabular}

\begin{tabular}{c|c|c} 
& co-quasi-idempotent element $\tau$ & weight $\gamma$ \\
\hline$\tau_{1}$ & $k_{1} f_{2}+k_{2} f_{3}+k_{3} f_{4}$ & $-k_{1}$ \\
\hline$\tau_{2}$ & $k_{1} f_{1}+k_{2} f_{3}+k_{3} f_{4}$ & $-k_{1}$ \\
\hline$\tau_{3}$ & $k_{1} f_{1}+k_{1} f_{2}$ & $-k_{1}$ \\
\hline$\tau_{4}$ & $k_{1} f_{3}+k_{2} f_{4}$ & 0
\end{tabular}

where $k_{i}, l_{j} \in \mathbb{C}, i, j=1,2,3$.

Next we assume that $k_{1} \neq 0$ and $l_{1} \neq 0$.

By [7, Corollary 2.4], if we set $l=\left(-2 l_{1}\right)^{-1}$, then the tridendriform algebra structures on $H_{4}$ are given by $\left(H_{4}, \prec_{i}, \succ_{i}, \mu_{H_{4}}\right), i=1,2,3$, where

\begin{tabular}{c|c|c|c|c}
$\prec_{1}$ & 1 & $x$ & $y$ & $x y$ \\
\hline 1 & $l \xi_{1}$ & $l\left(l_{1}(1+x)-l_{3} y-l_{2} x y\right)$ & $-\frac{1}{2}(y+x y)$ & $-\frac{1}{2}(y+x y)$ \\
\hline$x$ & $l\left(l_{1}(1+x)+l_{3} y+l_{2} x y\right)$ & $l\left(l_{1}(1+x)-l_{2} y-l_{3} x y\right)$ & $-\frac{1}{2}(y+x y)$ & $-\frac{1}{2}(y+x y)$ \\
\hline$y$ & $-\frac{1}{2}(y-x y)$ & $-\frac{1}{2}(y-x y)$ & 0 & 0 \\
\hline$x y$ & $\frac{1}{2}(y+x y)$ & $\frac{1}{2}(y+x y)$ & 0 & 0
\end{tabular}

\begin{tabular}{c|c|c|c|c}
$\succ_{1}$ & 1 & $x$ & $y$ & $x y$ \\
\hline 1 & $l \xi_{1}$ & $l\left(l_{1}(1+x)-l_{3} y-l_{2} x y\right)$ & $-\frac{1}{2}(y+x y)$ & $-\frac{1}{2}(y+x y)$ \\
\hline$x$ & $l\left(l_{1}(1+x)-l_{3} y-l_{2} x y\right)$ & $l \xi_{1}$ & $-\frac{1}{2}(y+x y)$ & $-\frac{1}{2}(y+x y)$ \\
\hline$y$ & $-\frac{1}{2}(y+x y)$ & $\frac{1}{2}(y+x y)$ & 0 & 0 \\
\hline$x y$ & $-\frac{1}{2}(y+x y)$ & $\frac{1}{2}(y+x y)$ & 0 & 0
\end{tabular}




\begin{tabular}{c|c|c|c|c}
$\prec_{2}$ & 1 & $x$ & $y$ & $x y$ \\
\hline 1 & $l \xi_{2}$ & $l\left(l_{1}(-1+x)-l_{3} y-l_{2} x y\right)$ & $-\frac{1}{2}(y-x y)$ & $-\frac{1}{2}(-y+x y)$ \\
\hline$x$ & $l\left(l_{1}(-1+x)+l_{3} y+l_{2} x y\right)$ & $l\left(l_{1}(1-x)-l_{2} y-l_{3} x y\right)$ & $\frac{1}{2}(y-x y)$ & $-\frac{1}{2}(y-x y)$ \\
\hline$y$ & $-\frac{1}{2}(y+x y)$ & $\frac{1}{2}(y+x y)$ & 0 & 0 \\
\hline$x y$ & $-\frac{1}{2}(y+x y)$ & $\frac{1}{2}(y+x y)$ & 0 & 0
\end{tabular}

\begin{tabular}{c|c|c|c|c}
$\succ_{2}$ & 1 & $x$ & $y$ & $x y$ \\
\hline 1 & $l \xi_{2}$ & $l\left(l_{1}(-1+x)-l_{3} y-l_{2} x y\right)$ & $-\frac{1}{2}(y-x y)$ & $\frac{1}{2}(y-x y)$ \\
\hline$x$ & $l\left(l_{1}(-1+x)-l_{3} y-l_{2} x y\right)$ & $l \xi_{2}$ & $\frac{1}{2}(y-x y)$ & $-\frac{1}{2}(y-x y)$ \\
\hline$y$ & $-\frac{1}{2}(y-x y)$ & $-\frac{1}{2}(y-x y)$ & 0 & 0 \\
\hline$x y$ & $\frac{1}{2}(y-x y)$ & $\frac{1}{2}(y-x y)$ & 0 & 0
\end{tabular}

and $\prec_{3}=\succ_{3}=\mu_{H_{4}}$.

By Proposition 3.6, if we set $k=\left(-k_{1}\right)^{-1}$, then the tridendriform coalgebra structures on $H_{4}$ are given by $\left(H_{4}, \Delta_{\prec j}, \Delta_{\succ j}, \Delta_{H_{4}}\right), j=1,2,3$, where

$$
\left|\begin{array}{c|c}
\Delta_{\prec 1}(1)=0 & \Delta_{\succ 1}(1)=0 \\
\Delta_{\prec 1}(x)=-x \otimes x & \Delta_{\succ 1}(x)=-x \otimes x \\
\Delta_{\prec 1}(y)=l k_{2} 1 \otimes x-y \otimes x & \Delta_{\succ 1}(y)=l k_{2} x \otimes x \\
\Delta_{\prec 1}(x y)=-x \otimes x y+l k_{3} x \otimes 1 & \Delta_{\succ 1}(x y)=-x \otimes x y-x y \otimes 1+l k_{3} 1 \otimes 1
\end{array}\right|
$$

$$
\begin{array}{c|c}
\Delta_{\prec 2}(1)=-1 \otimes 1 & \Delta_{\succ 2}(1)=-1 \otimes 1 \\
\Delta_{\prec 2}(x)=0 & \Delta_{\succ 2}(x)=0 \\
\Delta_{\prec 2}(y)=-1 \otimes y+l k_{2} 1 \otimes x & \Delta_{\succ 2}(y)=-1 \otimes y-y \otimes x+l k_{2} x \otimes x \\
\Delta_{\prec 2}(x y)=l k_{3} x \otimes 1-x y \otimes 1 & \Delta_{\succ 2}(x y)=l k_{3} 1 \otimes 1
\end{array}
$$

and

$$
\begin{aligned}
& \Delta_{\prec 3}(1)=\Delta_{\succ 3}(1)=-1 \otimes 1, \\
& \Delta_{\prec 3}(x)=\Delta_{\succ 3}(x)=-x \otimes x, \\
& \Delta_{\prec 3}(y)=\Delta_{\succ 3}(y)=-1 \otimes y-y \otimes x, \\
& \Delta_{\prec 3}(x y)=\Delta_{\succ 3}(x y)=-x \otimes x y-x y \otimes 1 .
\end{aligned}
$$

With notations above, then by Theorem 3.7, the tridendriform bialgebra structures on $H_{4}$ are given by $\left(H_{4}, \prec_{i}, \succ_{i}, \mu_{H_{4}}, \Delta_{\prec j}, \Delta_{\succ j}, \Delta_{H_{4}}\right), i, j=1,2,3$.

By [7, Prosition 2.2], $\left(H, R_{\xi_{i}}\right), i=1,2,3$ are Rota-Baxter algebras of weight $\lambda_{i}, i=$ $1,2,3$, where $\lambda_{1}=\lambda_{2}=-2 l_{1}, \lambda_{3}=-l_{1}$ and

\begin{tabular}{c|c|c|c|} 
& $R_{\xi_{1}}$ & $R_{\xi_{2}}$ & $R_{\xi_{3}}$ \\
\hline 1 & $\xi_{1}$ & $\xi_{2}$ & $\xi_{3}$ \\
\hline$x$ & $l_{1}(1+x)-l_{3} y-l_{2} x y$ & $l_{1}(-1+x)-l_{3} y-l_{2} x y$ & $l_{1} x$ \\
\hline$y$ & $l_{1}(y+x y)$ & $l_{1}(y-x y)$ & $l_{1} y$ \\
\hline$x y$ & $l_{1}(y+x y)$ & $l_{1}(-y+x y)$ & $l_{1} x y$
\end{tabular}.

By Proposition 3.8, $\left(H, Q_{\tau_{j}}\right), j=1,2,3,4$ are Rota-Baxter coalgebras of weight $\gamma_{j}, j=$ $1,2,3,4$, where $\gamma_{1}=\gamma_{2}=\gamma_{3}=-k_{1}, \gamma_{4}=0$ and

\begin{tabular}{c|c|c|c|c} 
& $Q_{\tau_{1}}$ & $Q_{\tau_{2}}$ & $Q_{\tau_{3}}$ & $Q_{\tau_{4}}$ \\
\hline 1 & 0 & $k_{1} 1$ & $k_{1} 1$ & 0 \\
\hline$x$ & $k_{1} x$ & 0 & $k_{1} x$ & 0 \\
\hline$y$ & $k_{2} x$ & $k_{1} y$ & $k_{1} y$ & 0 \\
\hline$x y$ & $k_{1} x y+k_{3} 1$ & $k_{3} 1$ & $k_{1} x y$ & $k_{2} 1$
\end{tabular}.


With notations above, then by Theorem $3.9,\left(H, R_{\xi_{i}}, Q_{\tau_{j}}\right), i=1,2,3, j=1,2,3,4$ are Rota-Baxter bialgebras of weight $\left(\lambda_{i}, \gamma_{j}\right), i=1,2,3, j=1,2,3,4$.

Acknowledgment. The authors are deeply indebted to the referees for their very useful suggestions and some improvements to the original manuscript. This work was partially supported by 2020 Research and innovation funding project for Postgraduates of Henan Normal University, Natural Science Foundation of Henan Province (No. 20A110019) and National Natural Science Foundation of China (No. 11801150). T. Ma is grateful to the Erasmus Mundus project FUSION for supporting the postdoctoral fellowship visiting to Mälardalen University, Västeras, Sweden and to the Division of Applied Mathematics at the School of Education, Culture and Communication for cordial hospitality.

\section{References}

[1] G. Baxter, An analytic problem whose solution follows from a simple algebraic identity, Pacific J. Math. 10, 731-742, 1960.

[2] L. Guo, An Introduction to Rota-Baxter Algebra, Surveys of Modern Mathematics, 4. International Press, Somerville, MA; Higher Education Press, Beijing, 2012.

[3] L. Guo, Properties of free Baxter algebras, Adv. Math. 151, 346-374, 2000.

[4] L. Guo and W. Keigher, Baxter algebras and shuffle products, Adv. Math. 150, 117$149,2000$.

[5] L. Guo and B. Zhang, Polylogarithms and multiple zeta values from free Rota-Baxter algebras, Sci. China Math. 53 (9), 2239-2258, 2010.

[6] L. Guo, J.-Y. Thibon and H. Yu, Weak composition quasi-symmetric functions, RotaBaxter algebras and Hopf algebras, Adv. Math. 344, 1-34, 2019.

[7] R.Q. Jian, Quasi-idempotent Rota-Baxter operators arising from quasi-idempotent elements, Lett. Math. Phys. 107, 367-374, 2017.

[8] R.Q. Jian and J. Zhang, Rota-Baxter coalgebras, arXiv:1409.3052.

[9] T.S. Ma and L.L. Liu, Rota-Baxter coalgebras and Rota-Baxter bialgebras, Linear Multilinear Algebra, 64 (5), 968-979, 2016.

[10] D.E. Radford, Hopf Algebras, KE Series on Knots and Everything, World Scientific, Vol. 49, New Jersey, 2012.

[11] G.C. Rota, Baxter algebras and combinatorial identities I, II, Bull. Amer. Math. Soc. 75 (2), 325-329, 330-334, 1969.

[12] E.J. Taft, The order of the antipode of finite dimensional Hopf algebra, Proc. Nat. Acad. Sci. USA. 68, 2631-2633, 1971. 